\title{
Inverse Optimality of Pure Proportional Navigation Guidance for Stationary Targets
}

\author{
Seokwon Lee * and Namhoon Cho ${ }^{\dagger}$ \\ Cranfield University, Cranfield, Bedfordshire, MK43 OAL, United Kingdom
}

\begin{abstract}
I. Introduction
Analytical examination of Proportional Navigation Guidance law (PNG) has received much attention from researchers as it is widely used for missile systems. The concept of PNG is to generate a turn rate command in proportion to the line-of-sight rate to form a stable collision geometry by nullifying the heading error defined with respect to the zero-effort-interception course. Among many variations of PNG, the Pure Proportional Navigation Guidance (PPNG) law which realises the heading error regulation through the command for lateral acceleration perpendicular to the pursuer's velocity vector is identified as the subject of major research thrusts since most tactical missiles steer through aerodynamic side force. The capturability of PPNG has been extensively studied [1, 2]. Also, various missile guidance laws meeting terminal constraints have been developed by taking PPNG as the baseline.

Characterisation of the optimality of the PNG holds importance in justification of its use as an optimal solution to the given design problems, hence, several approaches have been proposed. One approach is to analyse the performance of PNG by solving a forward optimal control problem with a predetermined performance index. The performance of the PNG has often been investigated in a linear setting by linearising the engagement kinematics around the collision course. The linearised version of PNG with the navigation gain $N=3$ was originally shown to be the optimal solution minimising control energy under the assumptions of constant closing speed and small perturbation from the collision course [3]. The linearised treatment was then extended to incorporate the acceleration response lag in [4] while the optimal solution begins to depart from the linearised form of the PNG due to additional terms. Later, [5] showed that PNG with an arbitrary positive navigation gain minimises a range-weighted control energy subject to the system equation re-defined into a linear form by change of variables. Recently, [6] provided a more general understanding about the optimality of pure PNG against a stationary target as it was shown to be the minimiser of a weighted $p$-norm of lateral acceleration for $p \in(1, \infty)$ with respect to the same re-defined linear system. Meanwhile, optimal solutions for the interception problem have been investigated with different forms of exact nonlinear formulations in [7-11]. Although the derivations differ slightly in detail, all of these studies provided basically the same physical understanding that the nonlinear optimal guidance solutions minimising the performance index given by either pure control energy or that

\footnotetext{
*Research Fellow, Centre for Autonomous and Cyber-Physical Systems, School of Aerospace, Transport and Manufacturing, seokwon.lee@cranfield.ac.uk, AIAA Member

${ }^{\dagger}$ Research Fellow, Centre for Autonomous and Cyber-Physical Systems, School of Aerospace, Transport and Manufacturing, n.cho@cranfield.ac.uk, AIAA Member
} 
combined with engagement duration closely resemble the behaviour of PNG when the initial heading error is small.

The optimality of guidance laws can also be studied from the inverse optimal control perspective. This approach is to obtain the necessary and sufficient condition for a given control law to be optimal. Kalman [12] first posed and partly solved the inverse problem of the optimal control theory determining the performance indices for which a given control law is optimal. Jameson and Kreindler [13] derived the necessary and sufficient condition for a multi-dimensional, time-varying, linear state-feedback policy to minimise a quadratic performance index. They remarked that the the state weighting matrix in performance index, $Q$, is not necessarily non-negative definite under certain conditions. Based on the result of [13], Kreindler [14] showed that PNG with any navigation gain $N \geq 2$ minimises a corresponding performance index. Likewise, Lee et al. [15] investigated the optimality of linear time-varying guidance laws for impact angle control through the associated inverse optimal control problem.

Despite extensive research efforts, the optimality characteristics of PNG is still not delineated to its entirety due mainly to the exclusion of nonzero state weighting in the performance index and the linearising approximation in the earlier studies. Most of the previous studies provided interpretations for the PNG as the minimiser of the weighted control energy performance indices. However, the formulation penalising only the input accounts for a limited region in the space of admissible navigation gain functions. In particular, the motivation for extending the class of allowed inverse optimal control formulations comes from the recent interests in the variants of the PNG that can enhance range observability in passive homing missiles. The observability enhancement objective typically demands a weaving-like manoeuvre pattern to induce variations in the line-of-sight rate [16-18]. The noticeable characteristic of the dual optimal control and estimation problem is that the design should trade off between two conflicting objectives; observability enhancement benefits from manoeuvre maximisation, whereas steering reduction benefits from manoeuvre minimisation. When representing the associated performance index by a weighted sum of two objectives, the weight imposed on the information maximisation goal takes a negative value. This way of cost definition leads to a family of optimal control problems with a negative state weighting which is an indefinite one lying outside the standard linear quadratic regulator formulation. The earlier studies such as [19, 21] addressed dual homing guidance based on the associated indefinite linear quadratic optimal control problems, however, they were developed by considering particular choices for the state and input weighting functions. The current state-of-the-art is still lacking a full characterisation of feasible formulations.

This Note investigates optimality of the PPNG from the perspective of inverse optimal control. To take advantages of the mathematical tractability while preserving the exactness of analysis, this study considers a linear system transformed from the nonlinear engagement kinematics which takes the range as the independent variable. In this way, this study obviates linearising approximations unlike the existing studies [12, 14, 15]. The necessary and sufficient condition of the state and control weighting functions that consist the quadratic performance index is determined based on the linear quadratic optimal control theory. The solution to the inverse optimal control problem defines a set of infinitely many possible combinations of the weightings that correspond to the given navigation gain function. For this reason, this 
study proposes a particular solution procedure based on decomposition of the navigation gain function into control minimising and information maximising components to facilitate physically meaningful interpretation of the analysis.

The main contribution of this study is the optimality analysis of the PPNG performed in full generality. The new theoretical findings can explain the result of the former analysis in which the PPNG is derived as the minimum effort solution [5] and also describe a comprehensive design framework including the observability-enhanced guidance laws developed for the dual homing guidance problem. Furthermore, this study provides several examples illustrating how the PPNG with various navigation gain functions can be understood as optimal control solutions.

\section{Preliminaries}

In this section, the problem formulation will revolve around a system model description that takes range $r$ as the independent variable instead of time $t$ assuming that $r$ is monotonically decreasing with respect to $t$.

\section{A. Engagement Kinematics Represented with Respect to Range}

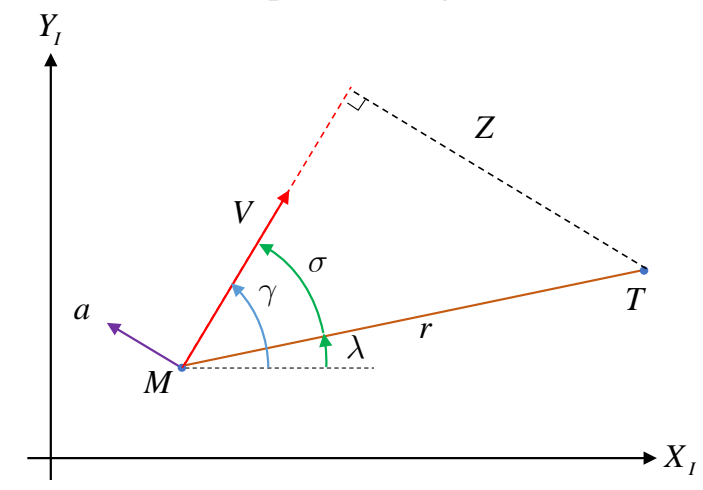

Fig. 1 Planar Engagement Guidance Geometry

Consider the planar engagement against a stationary target. Figure 1 shows the guidance geometry and the definition of variables. In Fig. 1 r is the range, $\lambda$ is the line-of-sight angle, $\gamma$ is the flight path angle, and $\sigma=\gamma-\lambda$ is the lead angle. Also, $V$ and $a$ denote the speed and the lateral acceleration, respectively. The planar engagement kinematics is given by

$$
\begin{aligned}
& \dot{r}=-V \cos \sigma \\
& \dot{\lambda}=-\frac{V}{r} \sin \sigma \\
& \dot{\gamma}=\frac{a}{V}
\end{aligned}
$$

Let $Z$ denote the zero-effort-miss, i.e., $Z=r \sin \sigma$. Also, suppose that $\dot{r}(t)<0$ where $\dot{(})=\frac{d}{d t}()$. Considering $Z$ 
and $r$ as the state variable and the independent variable, respectively, the state equation is given by

$$
Z^{\prime}(r)=-\frac{r}{V^{2}} a(r) \triangleq B(r) u(r)
$$

where ()$^{\prime}=\frac{d}{d r}(), B(r)=-\frac{r}{V^{2}}$, and $u=a$ is the input to the system. Note that the state equation, Eq. (2), is a linear range-varying system, which renders analysis of guidance laws easier. The dynamic system will evolve with monotonically decreasing variable $r$. The change of independent variable necessitates a special care in the treatment of the governing equation stating the necessary (and sufficient) condition for a control to be optimal, namely, the Hamilton-Jacobi-Bellman (HJB) equation. It is mainly because of the unusual order relationship between the lower and the upper limits in the performance index. The variant of HJB equation considering monotonically decreasing independent variable is presented in Appendix to avoid any unnecessary ambiguities.

\section{B. Characteristics of Pure Proportional Navigation Guidance}

From Eq. (2), the line-of-sight rate is closely related to the zero-effort-miss since $\dot{\lambda}=-\frac{V}{r^{2}} Z$. A state feedback control law with a range-varying gain $K(r)$ for the system in Eq. 2) can equivalently be understood as the PPNG with a range-varying navigation gain $N(r)$, because $\dot{\lambda}$ has a linear relation with $Z$. The following equation describes the feedback gain in relation to the navigation gain.

$$
u(r)=-K(r) Z(r)=K(r) \frac{r^{2}}{V} \dot{\lambda} \triangleq N(r) V \dot{\lambda}
$$

The closed-loop system is given by

$$
Z^{\prime}(r)=-B(r) K(r) Z(r)=\frac{r}{V^{2}} K(r) Z(r)=\frac{N(r)}{r} Z(r)
$$

and therefore, the state trajectory can be expressed as the transition function.

$$
Z(r)=Z\left(r_{0}\right) \exp \left[\int_{r_{0}}^{r} \frac{N(s)}{s} d s\right] \triangleq Z\left(r_{0}\right) \Phi\left(r, r_{0}\right)
$$

Without loss of generality, a given navigation gain function can always be represented in terms of its mean value and deviation as

$$
N(r)=N_{\text {avg }}+\tilde{N}(r) \text { where } N_{\text {avg }} \triangleq \frac{1}{r_{0}} \int_{0}^{r_{0}} N(s) d s
$$

Plugging the representation for navigation gain given by Eq. (6) into Eq. (5) and rearranging yields the closed-loop 
line-of-sight rate trajectory as

$$
\dot{\lambda}(r)=\dot{\lambda}\left(r_{0}\right) \exp \left[\int_{r_{0}}^{r} \frac{N(s)}{s} d s\right]=\dot{\lambda}\left(r_{0}\right)\left(\frac{r}{r_{0}}\right)^{N_{\text {avg }}-2} \exp \left[\int_{r_{0}}^{r} \frac{\tilde{N}(s)}{s} d s\right]
$$

The line-of-sight rate should be nullified at the end of engagement to hit the target while maintaining stability and robustness of the flight guidance and control system at the best possible level near the final time. The inverse analysis on the optimality of PPNG is physically meaningful only for the gains satisfying this basic requirement. In view of Eq. (7), the condition $\dot{\lambda}\left(r_{f}=0\right)=0$ requires the gain $N(r)$ to be a function such that $N_{\mathrm{avg}}>2$ and $\left|\exp \left[\int_{r_{0}}^{r} \frac{\tilde{N}(s)}{s} d s\right]\right|<\infty$.

\section{Inverse Optimality Analysis of Pure Proportional Navigation Guidance}

This section deals with the inverse optimality analysis of the PPNG. We first formulate the optimal control problem and derive the necessary and sufficient condition for the optimality according to the HJB equation A.8 in Appendix.A The analytic expressions of the performance index weightings will then be obtained for given PPNG structure. From the analytical results, we will present a relation between the navigation gain shaping and the pattern of the state weighting in the quadratic performance index. Giving considerations to nonzero and even indefinite state weighting in the analysis results in a more complete picture for the optimality of PPNG that includes optimal dual homing guidance laws [19-21]. Section III.C will investigate the characteristics of the performance index weightings associated with the navigation gain function. It will be shown that the solution to the inverse optimal control problem is not uniquely determined but composes a set of infinitely many combinations of weightings corresponding to the given navigation gain. It will also be shown in Sec. III.C that the navigation gain can be decomposed according to the physical meanings by decoupling the part of the gain pertaining to the input weighting from that associated with the state weighting. A way of specifying the solutions based on the navigation gain decomposition will be presented in Sec. III.D.

\section{A. Optimal Control Problem Formulation}

Consider the following optimal control problem formulation.

$$
\begin{aligned}
\text { minimise } \quad J & =\frac{1}{2} \int_{r_{f}=0}^{r_{0}}\left\{Q(s) Z^{2}(s)+R(s) u^{2}(s)\right\} d s \\
\text { subject to } \quad Z^{\prime}(r) & =B(r) u(r) \\
Z\left(r_{0}\right) & =Z_{0}, \quad Z\left(r_{f}=0\right)=0
\end{aligned}
$$

In view of the HJB equation given by Eq. A.8, we find that the Hamiltonian to be maximised is

$$
\mathcal{H}\left(Z, u, J_{Z}^{*}, r\right)=-\frac{1}{2}\left(Q(r) Z^{2}+R(r) u^{2}\right)+J_{Z}^{*} B(r) u
$$


Because the control is unconstrained, a necessary condition that the optimal control must satisfy is

$$
\left.\frac{\partial \mathcal{H}}{\partial u}\right|_{u^{*}}=-R(r) u^{*}+J_{Z}^{*} B(r)=0
$$

Observe that

$$
\frac{\partial^{2} \mathcal{H}}{\partial u^{2}}=-R(r)
$$

hence, if Eq. 10 is satisfied and $R>0$, it is sufficient to say that $u^{*}$ causes $\mathcal{H}$ to be a (local) maximum. From Eq. 10, we have

$$
u^{*}(r)=\frac{1}{R(r)} B(r) J_{Z}^{*}
$$

Substituting 12 into the HJB equation gives

$$
0=J_{r}^{*}-\frac{1}{2} Q(r) Z(r)^{2}+\frac{1}{2 R(r)}\left(B(r) J_{Z}^{*}\right)^{2}
$$

In the performance index, Eq. [8], the terminal cost is not considered since this study treats the final state as a fixed value. Consequentially, the boundary condition for the HJB equation is given by

$$
J^{*}\left(Z\left(r_{f}\right), r_{f}\right)=0 \text { on } Z\left(r_{f}=0\right)=0
$$

In order to solve the HJB equation, consider a cost-to-go function in the following form.

$$
J^{*}(Z(r), r)=\frac{1}{2} P(r) Z(r)^{2}
$$

Then, the derivatives of the cost-to-go function are

$$
J_{r}^{*}=\frac{1}{2} P^{\prime}(r) Z(r)^{2}, \quad J_{Z}^{*}=P(r) Z(r)
$$

Together with Eq. [15], the control law of Eq. [12] becomes

$$
u^{*}=\frac{1}{R(r)}(B(r) P(r)) Z(r)
$$

Also, substituting the guess solution given by Eq. (15) into Eq. (13) gives

$$
0=\frac{1}{2} P^{\prime}(r) Z(r)^{2}-\frac{1}{2} Q(r) Z(r)^{2}+\frac{1}{2 R(r)}(B(r) P(r))^{2} Z(r)^{2}
$$


Since Eq. 18 must be satisfied for all $Z(r)$, the following should be satisfied.

$$
0=P^{\prime}(r)-Q(r)+\frac{1}{R(r)}(B(r) P(r))^{2}
$$

Remark 1 (Closed-Form Solution for Forward Optimal Control Problem).

Although this study mainly concerns the inverse optimality of PPNG, a summary of the closed-form solution for the forward optimal control problem that results in a optimal guidance law of PPNG form is presented here for reference. The solution for the differential Riccati equation can be obtained for certain combinations of weighting function choices including the dual optimal control cases. The scalar Riccati differential equation associated with the general range-varying gain PPNG is given by Eq. [19]. If there exists a differentiable function $f(r)$ that relates the pair of weighting functions $(R(r), Q(r))$ as

$$
Q(r)=\frac{B^{2}(r)}{R(r)} f^{2}(r)-f^{\prime}(r)
$$

then $P_{0}(r)=f(r)$ is a particular solution of the Riccati equation, and the general solution can be written as

$$
P(r)=P_{0}(r)+g(r)\left[C-\int \frac{B^{2}(s)}{R(s)} g(s) d s\right]^{-1}
$$

where

$$
g(r) \triangleq \exp \left[\int 2 \frac{B^{2}(s)}{R(s)} P_{0}(s) d s\right]
$$

and $C$ is an arbitrary constant.

\section{B. Formulation Reconstruction for Inverse Optimality Analysis}

The main problem of inverse optimality analysis is to reconstruct the performance index about which the given control law, e.g., PPNG in this study, is optimal. In other words, the problem in this section is to find combinations of the functions $Q(r)$ and $R(r)$ when solved together for the optimal control yields the given feedback gain $K(r)$ (or, equivalently, the given navigation gain $N(r)$ ). A formal statement for the problem is as follows: "Given $K(r)$ (or $N(r)$ ) such that $Z\left(r_{f}\right)=0, \sigma\left(r_{f}\right)=0$, and $\dot{\lambda}\left(r_{f}\right)=0$, find the combination $(Q(r), R(r))$ that solve the inverse problem of linear quadratic optimal control."

Direct comparison between the given control law, i.e., PPNG, written as Eq. (3) and the form of the linear quadratic optimal control represented as Eq. 17] shows that

$$
K(r)=-\frac{1}{R(r)} B(r) P(r)
$$

Equation 23) clearly shows the coupling between $R(r)$ and $P(r)$, because $R(r)$ determines $P(r)$ uniquely for given 
$K(r)$ and $B(r)$. The sufficient condition for maximising the Hamiltonian discussed in the previous section using Eq. (11) implies that any scalar function $R(r)>0$ is admissible for our purpose. Therefore, it is reasonable to consider that $R(r)$ is specified as an arbitrary positive function. Then, function $P(r)$ that determines the optimal cost-to-go can be obtained as

$$
P(r)=-\frac{R(r) K(r)}{B(r)}=\frac{R(r) N(r) V^{4}}{r^{3}}
$$

The relationship in Eq. 24) is pointwise, i.e., holds for all $r$. The corresponding function $Q(r)$ can therefore be obtained by substituting Eq. 24] into Eq. (19) as

$$
\begin{aligned}
Q(r) & =P^{\prime}(r)+\frac{B(r)^{2}}{R(r)} P(r)^{2}=P^{\prime}(r)-B(r) K(r) P(r)=-\frac{d}{d r}\left(\frac{R(r) K(r)}{B(r)}\right)+R(r) K(r)^{2} \\
& =P^{\prime}(r)+\frac{B(r)^{2}}{R(r)} P(r)^{2}=P^{\prime}(r)+\frac{N(r)}{r} P(r)=\frac{d}{d r}\left(\frac{N(r) V^{4}}{r^{3}} R(r)\right)+\frac{N(r)^{2} V^{4}}{r^{4}} R(r)
\end{aligned}
$$

In this way, the pair $(P(r), Q(r))$ corresponding to a given $N(r)$ and a $R(r)$ which takes a strictly positive value for every $r$ can be determined.

Remark 2 (Multiplicity of Inverse Optimal Control Solutions).

It is worth noting that the necessary and sufficient condition for the inverse optimality itself does not specify certain input weighting but only requires it to be strictly positive for any $r$. Given a navigation gain $N(r)$, the inverse optimal control solutions can be determined in various ways depending on the choice of the input weighting $R(r)$. For example, if we choose one of the simplest forms for $R(r)$ as

$$
R(r)=r^{\rho}
$$

where $\rho$ is an arbitrary real constant, $(P, Q)$ can be obtained by using Eqs. 24 and 25) as

$$
\begin{gathered}
P(r)=N(r) V^{4} r^{\rho-3} \\
Q(r)=N(r) V^{4} r^{\rho-4}\left(\rho-3+N(r)+r \frac{N^{\prime}(r)}{N(r)}\right)
\end{gathered}
$$

Equations (26)-(28) indicate that there are infinitely many combinations of weights $(Q, R)$ differed by $\rho$ for a given navigation gain $N(r)$. For instance, if the navigation gain is given as $N(r)=3$, then $(Q, R)=(0,1)$ when $\rho=0$ and $(Q, R)=\left(-3 V^{4} r^{-5}, r^{-1}\right)$ when $\rho=-1$. 


\section{Characteristics of Weighting Functions and Decomposition of Navigation Gain}

In this section, we discuss the characteristics of the performance index weightings corresponding to the choice of the guidance gain of the PPNG. As discussed in Sec. III.A, the input weighting function $R(r)$ should be positive for maximum Hamiltonian optimality, Eq. [11, and the sign of $P(r)$ is directly related to the navigation gain from Eq. (24) as

$$
\operatorname{sign}(R(r))>0, \quad \operatorname{sign}(N(r))=\operatorname{sign}(P(r))
$$

On the contrary, $\operatorname{sign}(Q)$ has no direct relation with sign $(N)$. The function $Q(r)$ derived in Eq. (25) does not necessarily attain positive value for every $r$, because $P^{\prime}(r)$ can be negative for some $r$ depending on the variation of function $K(r)$ (or $N(r)$ ) along $r$. This relation indicates that the value of function $N(r)$ can be positive at some $r$ while the function $Q(r)$ takes a negative value at that point.

In this aspect, the analytical results of this study provide a more generalised formulation that is useful for the optimal dual homing guidance designs. The navigation gain $N(r)$ should change its sign along $r$ several times for a guidance law of the PPNG form to issue a steering acceleration command with alternating sign. The sign change of $N(r)$ is permissible as long as the average value $N_{\text {avg }}$ satisfies the condition stated in Sec. III.B for final constraint satisfaction. The function $P(r)$ that defines the optimal cost-to-go as in Eq. (15) will inevitably change its sign since sign $(P(r))=\operatorname{sign}(N(r))$.

Let us consider the control law given by Eq. (3) to see the qualitative understandings about the weighting functions and the navigation gain more clearly. By substituting Eq. (5) into the performance index defined in Eq. (8) with Eq. (3), the cost-to-go can be represented as

$$
J(Z(r), r, u(r))=\frac{1}{2} \int_{0}^{r}\left\{Q(s)+R(s) K^{2}(s)\right\} \Phi^{2}\left(s, r_{0}\right) d s Z^{2}\left(r_{0}\right)
$$

The optimal (minimum) cost-to-go can be written by substituting Eq. (5) into Eq. (15) as

$$
J^{*}(Z(r), r)=\frac{1}{2} P(r) Z^{2}(r)=\frac{1}{2} P(r) \Phi^{2}\left(r, r_{0}\right) Z^{2}\left(r_{0}\right)
$$

for the tuple $(P, Q, R)$ satisfying Eq. 19]. The minimum cost-to-go is the cost-to-go evaluated with $K$ given by Eq. 23],

$\left.J(Z(r), r, u(r))\right|_{K=-\frac{B P}{R}}$, where $P(r)$ satisfies Eq. [19]. Equating the right-hand sides of Eqs. 30] and 31] shows that $P(r)$ can be represented as a sum of two separate functions, that is,

$$
P(r)=\frac{1}{\Phi^{2}\left(r, r_{0}\right)} \int_{0}^{r} Q(s) \Phi^{2}\left(s, r_{0}\right) d s+\frac{1}{\Phi^{2}\left(r, r_{0}\right)} \int_{0}^{r} R(s) K^{2}(s) \Phi^{2}\left(s, r_{0}\right) d s \triangleq P_{\max \text { info }}(r)+P_{\min \text { ctrl }}(r)
$$

where the functions $P_{\max \text { info }}(r)$ and $P_{\min }$ ctrl $(r)$ pertain to the information maximisation and the manoeuvre minimisation objectives, respectively, and they are defined in the order as shown in Eq. 32. Because the navigation gain $N$ is linearly 
related to $P(r)$ as shown in Eq. (24), $N(r)$ can also be decomposed as

$$
N(r)=\frac{r^{3}}{R(r) V^{4}} P_{\max \text { info }}(r)+\frac{r^{3}}{R(r) V^{4}} P_{\min \text { ctrl }}(r) \triangleq N_{\max \text { info }}(r)+N_{\text {min ctrl }}(r)
$$

Equation (32) shows that $P_{\max \text { info }}(r)$ can attain negative values depending on the design of $Q(r)$ while $P_{\text {min ctrl }}(r)$ is always positive. If the state weighting function $Q(r)$ is negative for all $r$, then $P_{\max \text { info }}(r)$ is negative for all $r$. In this case, $P(r)$ becomes positive if $\left|P_{\max \text { info }}(r)\right|<P_{\min \text { ctrl }}(r)$ and negative if $\left|P_{\max \text { info }}(r)\right|>P_{\min \text { ctrl }}(r)$.

\section{Specific Determination of Inverse Optimal Control Solutions}

Section III.B showed how the weighting $Q(r)$ can be determined by the navigation gain $N$ when the input weighting $R(r)$ is given, and the inverse optimal control solutions can be determined in various ways depending on the choice of the input weighting $R(r)$ given a navigation gain $N(r)$. It is preferable to avoid arbitrary definition of the input weighting for more systematic determination of the inverse optimal control solution. To this end, this study develops a specific solution determination method based on the physically meaningful decomposition of the navigation gain $N(r)=N_{\min \text { ctrl }}+N_{\max \text { info }}$ discussed in Sec. III.C to assign a particular pair $(Q, R)$. The subsequent sections present how the tuple $(P, Q, R)$ representing the minimised performance index can be obtained for both the cases with and without the state weighting $Q(r)$.

\section{Case 1. Inverse Optimal Control Solution Considering Only Input Weighting}

Let us investigate the optimality of PPNG when $N(r)$ is designed only for input minimisation, i.e., $N_{\max \text { info }}=0$. In this case, the influence of state on the performance index is ignored, i.e., $Q=0$. From Eq. (25), $N(r)$ can be represented in terms of $P(r)$ as

$$
N(r)=-r \frac{P^{\prime}(r)}{P(r)}
$$

For any positive function $N>0, P$ must satisfy the monotonic relation as

$$
P(r)>0 \text { and } \quad P^{\prime}(r)<0
$$

Then, $P$ can be expressed for some positive constant $P_{0}$ as

$$
P(r)=P_{0} \exp \left[-\int_{r_{0}}^{r} \frac{N(s)}{s} d s\right]=\frac{P_{0}}{\Phi\left(r, r_{0}\right)}=P_{0} \Phi\left(r_{0}, r\right)
$$

Likewise, the input weighting function $R$ associated with $P$ of Eq. 36 can be written as

$$
R(r)=\frac{P_{0} r^{3}}{V^{4} N(r)} \exp \left[-\int_{r_{0}}^{r} \frac{N(s)}{s} d s\right]=\frac{P_{0} r^{3}}{V^{4} N(r)} \Phi\left(r_{0}, r\right)
$$


Equation (37) is exactly the relation shown in the previous study [5] which clarified the minimum effort optimality of PPNG in the stationary target case based on a nonlinear formulation. Considering the constant gain $N, P(r)$ and $R(r)$ can be simplified as

$$
P(r)=P_{0} r_{0} \times r^{-N}, \quad R(r)=\frac{P_{0} r_{0}}{V^{4} N} r^{-(N-3)}
$$

\section{Case 2. Inverse Optimal Control Solution Considering Both State and Input Weightings}

Consider the navigation gain that can be decomposed into the additive form as $N(r)=N_{\min \text { ctrl }}(r)+N_{\max \text { info }}(r)$. Then, we may assign the functions $(P, Q, R)$ from the homogeneous solution obtained from previous section. That is, $P_{\min c t r l}$ and $N_{\min c t r l}$ are thought to satisfy

$$
P_{\min c t r l}^{\prime}+\frac{N_{\min c t r l}}{r} P_{\min c t r l}=0
$$

which is identical in its form to Eq. (34) and has $P_{\min c t r l}(r)=P_{0} \exp \left[-\int_{r_{0}}^{r} \frac{N_{\min c t r l}(s)}{s} d s\right]$ for some $P_{0}>0$ as its solution. From Eq. [33, $R(r)$ and $P_{\max \text { info }}(r)$ can be obtained as

$$
\begin{gathered}
R(r)=\frac{r^{3}}{N_{\text {min ctrl }}(r) V^{4}} P_{\operatorname{minctrl}}(r)=\frac{P_{0} r^{3}}{N_{\min \text { ctrl }}(r) V^{4}} \exp \left[-\int_{r_{0}}^{r} \frac{N_{\min \text { ctrl }}(s)}{s} d s\right] \\
P_{\text {max info }}(r)=N_{\text {max info }}(r) \frac{R(r) V^{4}}{r^{3}}=P_{0}\left(\frac{N(r)}{N_{\min \text { ctrl }}(r)}-1\right) \exp \left[-\int_{r_{0}}^{r} \frac{N_{\text {min ctrl }}(s)}{s} d s\right]
\end{gathered}
$$

$Q(r)$ is also obtained as

$$
Q(r)=\frac{d}{d r}\left(\frac{N(r) V^{4}}{r^{3}} R(r)\right)+\frac{N(r)^{2} V^{4}}{r^{4}} R(r)
$$

Remark 3 (Multiplicity of Inverse Optimal Control Solutions with regard to partitioned $N$ ).

Partition of a given $N$ by two mutually dependent components $N_{\min \text { ctrl }}$ and $N_{\max \text { info }}$ is not unique. The part $N_{\min \text { ctrl }}$ might be taken fairly arbitrarily while the other part $N_{\text {max info }}$ is determined uniquely by the choice of $N_{\min \text { ctrl }}$. For example, one may consider the average $N_{\text {avg }}$ of a given navigation gain as the component $N_{\min \text { ctrl }}$ associated with mimisation of control effort; another can choose $N_{\min \text { ctrl }}$ as a range-dependent function. As a consequence of the multiplicity of partitions admissible for a fixed navigation gain $N$, the present analysis does not uniquely determine the solution pair $(Q, R)$.

\section{Examples}

This section gives illustrative examples of the inverse optimality analysis for the PPNG with particular types of navigation gain functions. Example 1 in Sec. IV.A presents an inverse optimal control solution corresponding to a polynomial navigation gain, considering performance index of the form $J=\int_{r_{0}}^{r_{f}} R(r) u^{2}(r) d r$ based on the discussion 
of Sec. III.D.1. By applying the development of Sec. III.D.2, examples 2 and 3 in Sec. IV.B and IV.C deal with the switching and alternating gains to investigate the solutions involving both state and input weightings in the performance index as in $J=\frac{1}{2} \int_{r_{0}}^{r_{f}}\left\{Q(r) Z^{2}(r)+R(r) u^{2}(r)\right\} d r$.

\section{A. Example 1: PPNG with Polynomial Navigation Gain}

The guidance gain is considered as

$$
N(r)=\sum_{i=0}^{n} N_{i} r^{i}=N_{0}+N_{1} r+\cdots+N_{n} r^{n}
$$

$(P, R)$ can be expressed in the polynomial gain as

$$
P(r)=P_{0}\left(\frac{r}{r_{0}}\right)^{-N_{0}} \exp \left(\sum_{i=1}^{n}-N_{i}\left(r^{i}-r_{0}^{i}\right)\right), \quad R(r)=\frac{P_{0} r_{0}^{3}}{\left(\sum_{i=0}^{n} N_{i} r^{i}\right) V^{4}}\left(\frac{r}{r_{0}}\right)^{-N_{0}+3} \exp \left(\sum_{i=1}^{n}-N_{i}\left(r^{i}-r_{0}^{i}\right)\right)
$$

\section{B. Example 2: Switched-Gain PPNG for Field-of-View Constrained Interception}

The guidance gain is considered as

$$
N= \begin{cases}N_{i}(=1) & r_{s w} \leq r \leq r_{0} \\ N_{f}(=3) & 0 \leq r \leq r_{s w}\end{cases}
$$

where $N_{f}=3$ is for energy optimal performance, and $N_{i}=1$ for maintaining constant look angle. Let us choose $N_{\text {min ctrl }}=N_{f}=3$ so that $N_{\max \text { info }}$ can be expressed as

$$
N_{\text {maxinfo }}=\left\{\begin{array}{cl}
-2 & r_{s w} \leq r \leq r_{0} \\
0 & 0 \leq r \leq r_{s w}
\end{array}\right.
$$

$P_{\min c t r l}$ and $R$ can be obtained from Eqs. (39) and (40) as

$$
P_{\text {min ctrl }}(r)=P_{\min c t r l}\left(r_{0}\right) \exp \left(\int_{r_{0}}^{r}-\frac{N_{f}}{s} d s\right)=P_{0}\left(\frac{r}{r_{0}}\right)^{-3}, \quad R=\frac{r^{3}}{N_{f} V^{4}} P_{\min c t r l}=\frac{r_{0}^{3} P_{0}}{3 V^{4}}
$$

$Q$ can be obtained by substituting $R$ obtained in Eq. 477 into Eq. (42) as

$$
Q(r)=\frac{N V^{4}}{r^{4}} R(N-3)=\left\{\begin{array}{cl}
-\frac{2 P_{0}}{3 r_{0}}\left(\frac{r_{0}}{r}\right)^{4} & r_{s w} \leq r \leq r_{0} \\
0 & 0 \leq r \leq r_{s w}
\end{array}\right.
$$




$$
P_{\text {maxinfo }}(r)=N_{\text {maxinfo }} \frac{R(r) V^{4}}{r^{3}}=\left\{\begin{array}{cc}
-\frac{2 P_{0}}{3}\left(\frac{r_{0}}{r}\right)^{3} & r_{s w} \leq r \leq r_{0} \\
0 & 0 \leq r \leq r_{s w}
\end{array}\right.
$$

\section{Example 3: PPNG for Observability Enhancement}

In this example, we consider the navigation gain in the decomposed form as shown in Eq. 333. A natural choice for analysing the problem with dual control objectives is to assume the presence of weighting for state as well as input.

\section{Case 1. Sinusoidal Navigation Gain}

The navigation gain is considered as

$$
N(r)=N_{c}+k \sin (a r)
$$

where $N_{c}$ is the constant central value of $N$, and $(k, a)$ represent the amplitude and frequency of the perturbed gain, respectively. Suppose that $N_{\min \text { ctrl }}$ is selected as $N_{c}$, then the corresponding $P_{\min c t r l}$ and $R$ can be obtained from Eqs. (39) and (40) as

$$
P_{\text {minctrl }}(r)=P_{\operatorname{minctrl}}\left(r_{0}\right) \exp \left(\int_{r_{0}}^{r}-\frac{N_{c}}{s} d s\right)=P_{0}\left(\frac{r}{r_{0}}\right)^{-N_{c}}, \quad R(r)=\frac{r^{3}}{N_{c} V^{4}} P_{\min c t r l}=\frac{r_{0}{ }^{3} P_{0}}{N_{c} V^{4}}\left(\frac{r}{r_{0}}\right)^{-\left(N_{c}-3\right)}
$$

$P_{\max \text { info }}$ and the state weighting $Q$ can be obtained according to Eqs. (41) and (42) as

$$
P_{\max \text { info }}(r)=\frac{k P_{0}}{N_{c}}\left(\frac{r}{r_{0}}\right)^{-N_{c}} \sin (\text { ar }), \quad Q(r)=R(r)\left(\frac{r}{V}\right)^{-4}\left(k^{2} \sin ^{2}(a r)+N_{c} k \sin (\operatorname{ar})+k a r \cos (a r)\right)
$$

\section{Case 2. Navigation Gain in Ref. [21]}

As a generalisation of the PPNG variant presented in Ref. [21], consider the navigation gain given as

$$
N\left(t_{g o}\right)=\omega t_{g o} N_{f} \cot \left(\frac{\omega t_{g o} N_{f}}{N_{f}}\right)
$$

where $t_{g o}$ is approximated by $t_{g o} \approx \frac{r}{V}$ and $N_{f}>1$ is a constant. Note that $\lim _{r \rightarrow 0} N(r)=N_{f}$.

By decomposing the navigation gain into two separate parts, $N_{\min c t r l}$ and $N_{\max \text { info }}$ can be assigned as

$$
N_{\text {min ctrl }}=N_{f}, \quad N_{\text {max info }}=\omega\left(\frac{r}{V}\right)^{N_{f}} \cot \left(\frac{\omega}{N_{f}}\left(\frac{r}{V}\right)^{N_{f}}\right)-N_{f}
$$


Now, $P_{\min \text { ctrl }}$ and $R$ can be obtained by the substitution of $N_{f}$ into Eq. [51] as

$$
P_{\text {minctrl }}(r)=P_{0}\left(\frac{r}{r_{0}}\right)^{-N_{f}}, \quad R(r)=\frac{r_{0}^{3} P_{0}}{N_{f} V^{4}}\left(\frac{r}{r_{0}}\right)^{-\left(N_{f}-3\right)}
$$

Equation (55) shows that the input weighting is a power function of $r . Q$ and $P_{\max \text { info }}$ can be obtained as

$$
Q(r)=-R(r)\left(\omega\left(\frac{r}{V}\right)^{N_{f}-2}\right)^{2}, \quad P_{\operatorname{maxinfo}}(r)=\frac{P_{0}}{N_{f}}\left(\omega\left(\frac{r}{V}\right)^{N_{f}} \cot \left(\frac{\omega}{N_{f}}\left(\frac{r}{V}\right)^{N_{f}}\right)-N_{f}\right)\left(\frac{r}{r_{0}}\right)^{-N_{f}}
$$

One can observe from Eq. [56] that the state weighting turns out to be a power function of $\frac{r}{V}$, which is the simple approximation for $t_{g o}$. In Ref. [21], $N_{f}$ was chosen as 3 for a particular case. In a way similar to the previous cases, the pair $(R, Q)$ can be obtained as

$$
R=\frac{P_{0} r_{0}^{3}}{3 V^{4}}, \quad Q(r)=-R\left(\omega \frac{r}{V}\right)^{2}
$$

which is identical to the performance index in Ref. [21]. Note that the input weighting is given by a constant when $N_{f}=3$. In this particular case, $\left(P_{\min c t r l}, P_{\max \text { info }}\right)$ can be expressed as

$$
P_{\min , \mathrm{ctrl}}(r)=P_{0}\left(\frac{r}{r_{0}}\right)^{-3}, \quad P_{\max \text { info }}(r)=\frac{P_{0}}{3}\left(\omega\left(\frac{r}{V}\right)^{3} \cot \left(\frac{\omega}{3}\left(\frac{r}{V}\right)^{3}\right)-3\right)\left(\frac{r}{r_{0}}\right)^{-3}
$$

\section{Numerical Simulation}

This section illustrates the analytical understandings provided by this study through numerical simulation. The examples of the PPNG with various range-dependent navigation gain functions introduced in the previous section are considered for simulation. In all simulation cases, the missile speed and the initial flight path angle are $V=300 \mathrm{~m} / \mathrm{s}$ and $\gamma_{0}=30 \mathrm{deg}$, respectively. The initial distance and line-of-sight angle measured with respect to the target are $r_{0}=10,000 \mathrm{~m}$ and $\lambda_{0}=0 \mathrm{deg}$, respectively. Simulation is terminated if the range decreases below $0.1 \mathrm{~m}$.

\section{A. Example 1}

In this example, the navigation gain functions belonging to the polynomial family are considered to exemplify how the analysis of inverse optimality applies to an actual design. Polynomials of degree 1 and 2 that satisfy the boundary conditions $N(0)=3$ and $N\left(r_{0}\right)=1$ are considered. A standard case with the constant navigation gain of $N=3$ is also included in the simulation to compare the solution characteristics.

Figure 2 shows the simulation result for Example 1. Navigation gain given by the linear and quadratic polynomials attains value less than the constant $N=3$ in the interval $r \in\left[0, r_{0}\right]$, which allows to elongate the flight path. Regardless of the type and the degree of function being used, zero-effort-miss decreases consistently as shown in Fig. $2 b$ as the navigation gain is continuously taking positive values. However, the choice of navigation gain directly affects the 


\section{Table 1 Simulation Parameters: Examples 2 and 3}

\begin{tabular}{lccccc}
\hline \hline Parameter & $r_{s w}$ & $N_{c}$ & $\omega$ & $a$ & $k$ \\
\hline Values & 3300 & 3 & 0.002 & $\frac{\pi}{6 r_{0}}$ & -8 \\
\hline \hline
\end{tabular}

acceleration response.

The solution behaviours depending on the navigation gain can be understood from the inverse optimal control perspective. Figure $2 \mathrm{~d}$ shows the Riccati solution $P$ and the input weight $R$ solved for each corresponding navigation gain $N$ by using Eq. (44). The solution $P$ tends to infinity as the missile approaches the target. This is compliant with the physical understanding that the fixed final state boundary condition for interception imposes an infinity weight on the final state in the performance index. Also, the input responses produced with the polynomial gains involve rapid manoeuvres in the vicinity of the final time. The input weight obtained as the inverse optimal control solution accounts for the trend; it is greater than one at the beginning of the engagement and decreases to zero at the end. This example clearly shows that the analysis done in the context of inverse optimal control provides consistent and reasonable understandings about the trajectory pattern governed by the range-dependent navigation gain.

\section{B. Examples 2 and 3}

The navigation gains developed specifically for field-of-view constrained interception in Example 2 and for observability enhancement in Example 3 are considered in the next example. In this simulation, the navigation gain is decomposed into information maximisation and control minimisation components where $N_{\text {min,ctrl }}$ is set to 3 . The switching navigation gain of Example 2 is set to be 1 in the first phase. The parameter $P_{0}$ is set to be $P_{0}=3 V^{4} / r_{0}{ }^{3}$ to normalise the input weight as $R=1$. With the setup fixing the input weight, this example focuses on the effects of the state weight $Q$ and the components comprising the Riccati solution $P_{\min , \mathrm{ctrl}}$ and $P_{\max \text {,info }}$ on the guidance performance. Other simulation parameters are summarised in Table 1

Figure 3 shows the simulation results. The fixed input weight $R$ represents the action of minimising control energy uniformly over the entire engagement, while the variation in the state weight $Q$ stands for the action of acquiring information relevant to the state variable with a different pattern. With regard to the switching navigation gain of Example 2, the state weight $Q$ takes a negative value before switching and follows the overall trend of $P_{\text {max,info }}$ which grows in its magnitude in the first phase as shown in Figs. 3e and 3f This tendency is mainly attributed to the trajectory intended to hold a constant look angle in the first phase while delaying regulation of heading error, which can be described with a negative state weight as it is conflicting with the minimisation of control effort over entire engagement. The constant navigation gain $N=3$ after switching allows for the input optimal performance over the remaining interval.

On the other hand, the observability-enhancement guidance laws described in Example 3 exhibit considerable shaping of the trajectory and require more control efforts due to the use of the alternating navigation gains. Figures 
3e and 3f show that the component $P_{\min \text {,ctrl }}$ takes a positive value for each $r$ with the input weight $R$ being positive, whereas $P_{\text {max,info }}$ takes a negative value for each $r$ with the state weight $Q$ also being negative.

The overall contribution of information maximising action is relatively small as compared to the minimisation of control effort, which implies that the latter governs the general trend thus leading to target interception. Nonetheless, information maximisation can be dominant in some region of $r$ where a negative navigation gain leads to growth in the heading error which in turn improves the degree of observability at the cost of delayed convergence to a collision course. A notable point is that the theoretical findings of this study is general enough to allow the navigation gain to be less than zero in some interval as long as it satisfies the feasibility condition discussed in Sec. II.B. Hence, the knowledge applies also to the dual homing guidance laws in the form of PPNG. The inverse optimality analysis performed in this study provides a fully comprehensive framework extending beyond the previous understanding that has been confined to the traditional perspective interpreting PPNG as the minimiser of control energy.

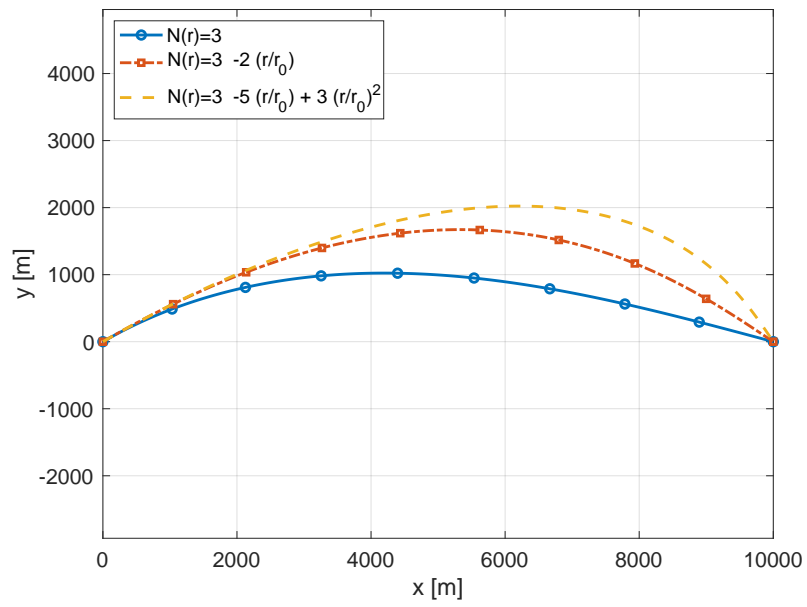

(a) Two-Dimensional Trajectory

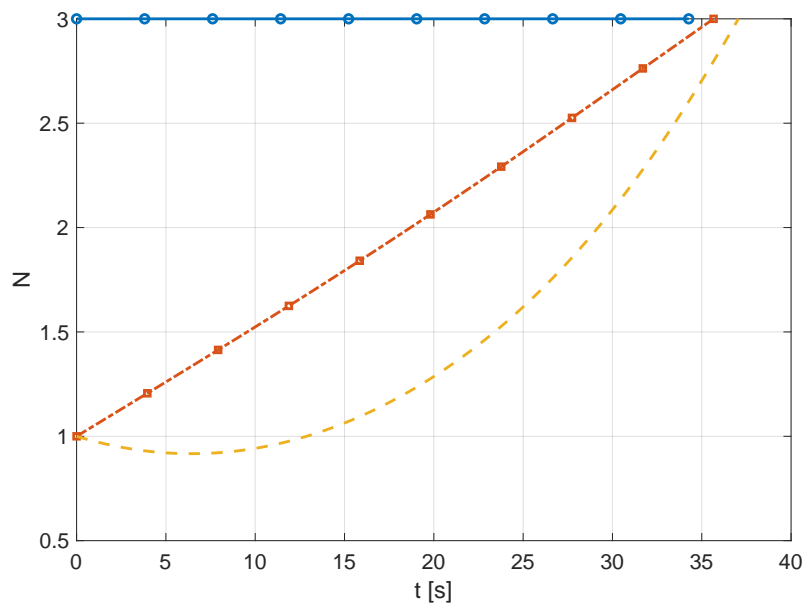

(c) Navigation Gain
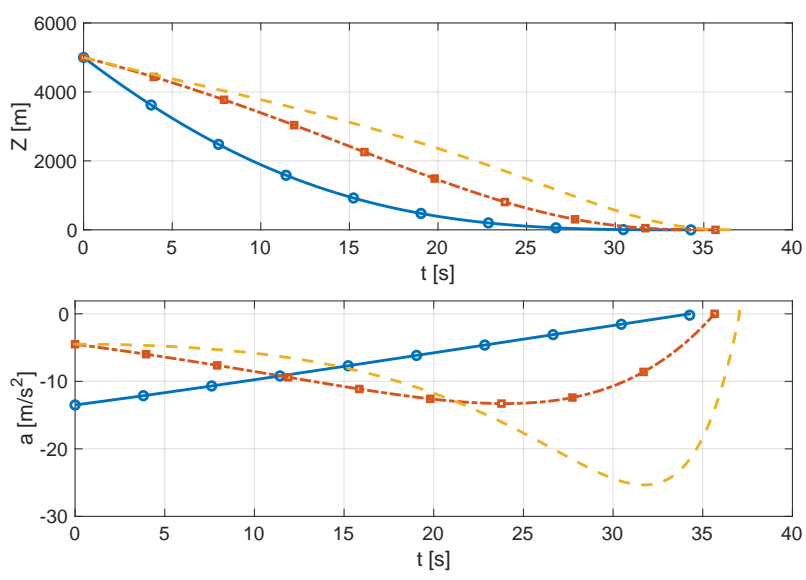

(b) Zero-Effort-Miss and Acceleration
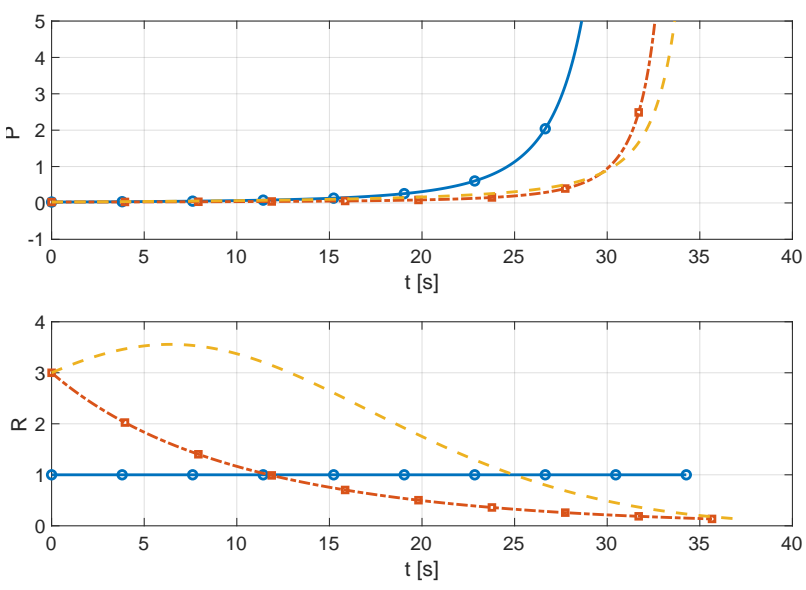

(d) Riccati Solution $P$ and Input Weight $R$

Fig. 2 Example 1: Polynomial Navigation Gain 


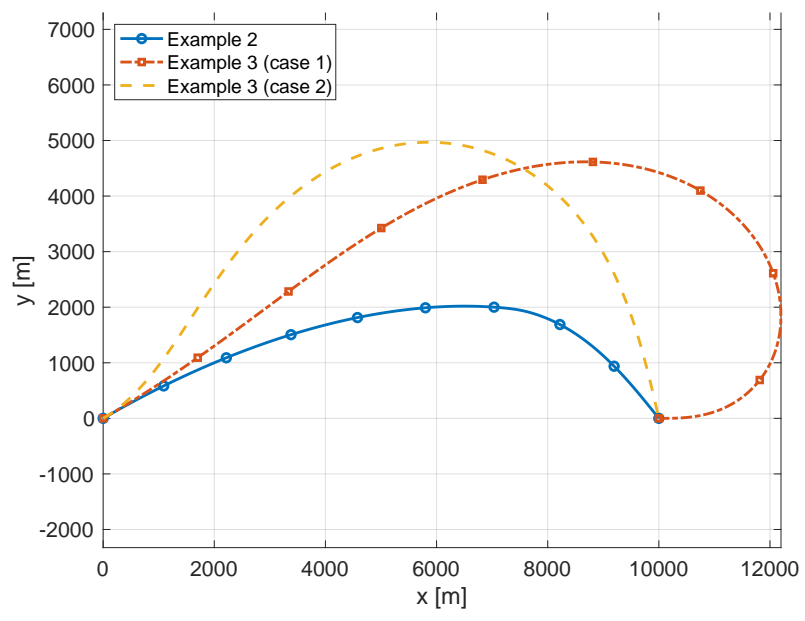

(a) Two-Dimensional Trajectory
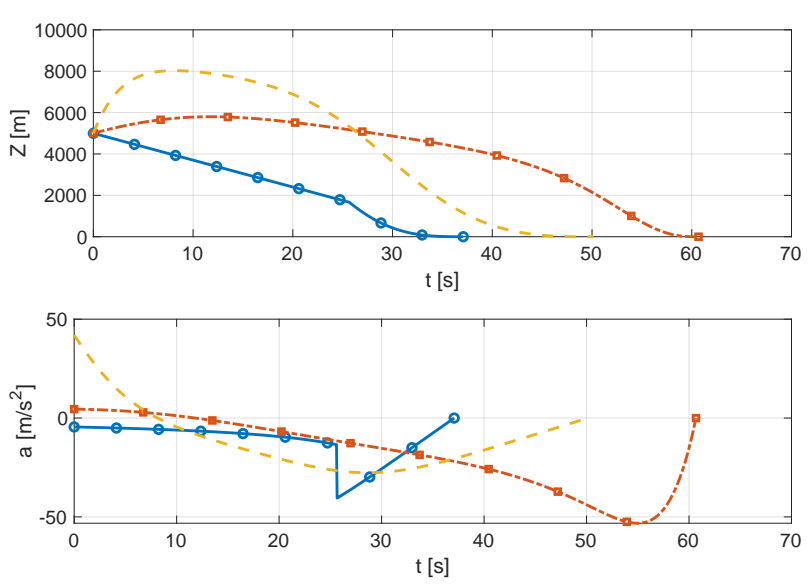

(c) Zero-Effort-Miss and Acceleration
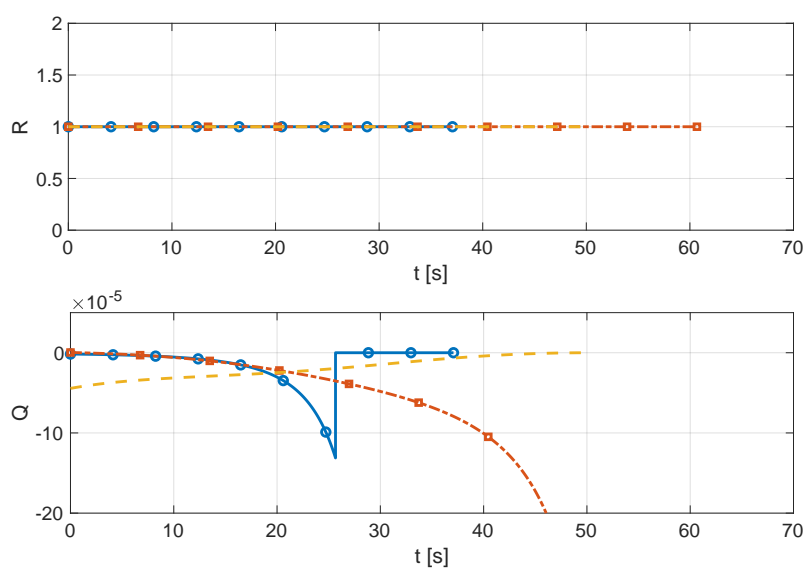

(e) Input Weight $R$ and State Weight $Q$

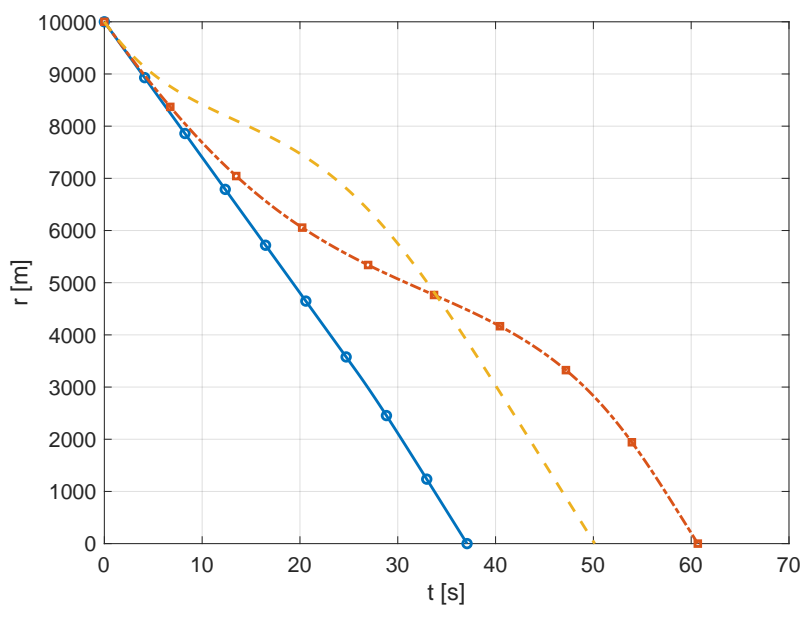

(b) Range

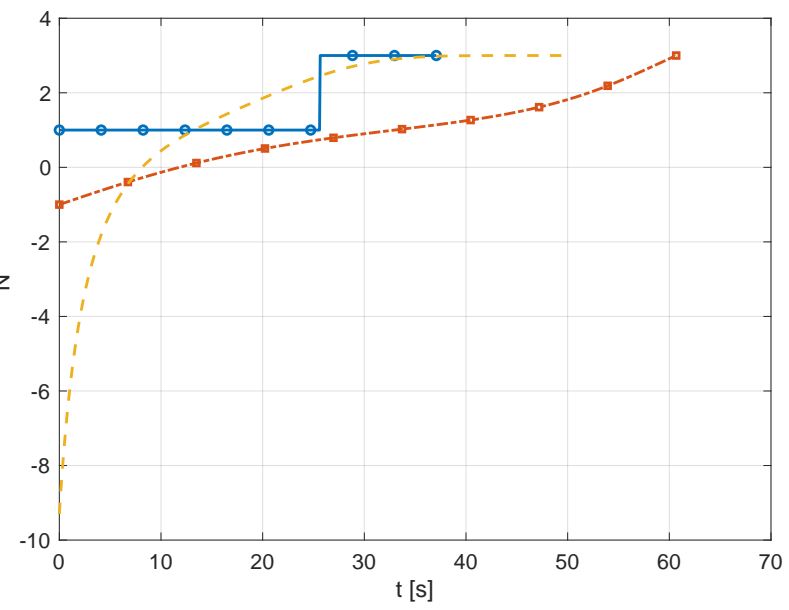

(d) Navigation Gain
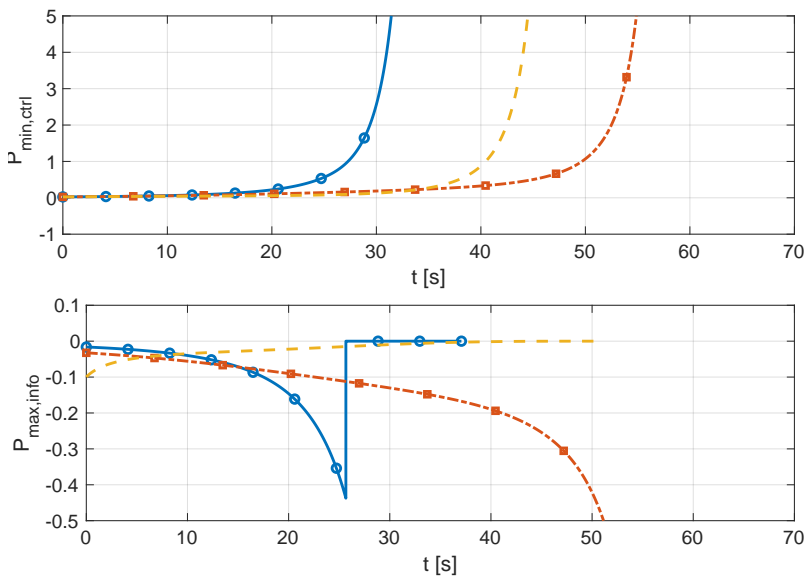

(f) Riccati Solution $P$

Fig. 3 Examples 2 and 3: Switching and Alternating Navigation Gains 


\section{Conclusion}

Optimality of the pure proportional navigation guidance law was investigated from the inverse optimal control perspective. The relationship between the navigation gain and the weightings included in the quadratic performance index was investigated by solving the inverse optimal problem. The optimality of the pure proportional navigation guidance law was analysed in more thoroughly with full generality compared with the relevant earlier studies. Notably, the state weighting can be indefinite while the regular condition on the input weighting is preserved, hence, the dual action due to two conflicting objectives can be imposed through the state weighting function. The practical value of this study is that the generalised analysis enables explaining the optimality of the family of pure proportional navigation guidance laws developed for the dual homing guidance problem which demands observability enhancement during the homing phase. Designers may utilise the result of this study either as a method of determining the performance index corresponding to a given navigation gain or as a complete procedure for linear quadratic optimal design of the navigation gain including the indefinite formulations.

\section{Appendix}

\section{A. Hamilton-Jacobi-Bellman Equation for Continuous-Time Optimal Control of Dynamic Systems with Monotonically Decreasing Independent Variable}

Consider the dynamic system with $r$ as the monotonically decreasing independent variable

$$
\mathbf{x}^{\prime}(r)=\mathbf{f}(\mathbf{x}(r), \mathbf{u}(r), r)
$$

and the performance index to be minimised by an optimal control

$$
J(\mathbf{x}(r), r, \mathbf{u}(r))=h\left(\mathbf{x}\left(r_{f}\right), r_{f}\right)+\int_{r_{f}}^{r} g(\mathbf{x}(s), \mathbf{u}(s), s) d s
$$

where $h$ and $g$ are specified functions. Let $r_{0}, r_{f}$, and $\mathbf{x}_{0}$ are given. Notice here that $r_{0} \geq r_{f}$. The problem can be formulated as follows: minimise $J$ given by Eq. A.2, subject to i) the differential equation given by Eq. A.1, ii) $\mathbf{x}\left(r_{0}\right)=\mathbf{x}_{0}$, iii) the set of terminal conditions $\mathbf{m}\left(\mathbf{x}\left(r_{f}\right), r_{f}\right)=0$, and iv) the set of admissible controls $\mathbf{u}(s) \in \mathcal{U}$.

Consider first the cost over the interval between $r$ and $r_{f}$, where $r_{f} \leq r$, of any control sequence $\mathbf{u}(s)$ in $r_{f} \leq s \leq r$. The optimal cost-to-go function, i.e., the value function, is then given by

$$
J^{*}(\mathbf{x}(r), r)=\min _{\substack{\mathbf{u}(s) \in \mathcal{U} \\ r_{f} \leq s \leq r}} J(\mathbf{x}(r), r, \mathbf{u}(r))
$$

By subdividing the interval and then writing explicitly the minimisation operation over the two subintervals, Eq. 
(A.3) can be rewritten as

$$
\begin{aligned}
J^{*}(\mathbf{x}(r), r) & =\min _{\substack{\mathbf{u}(s) \in \mathcal{U} \\
r_{f} \leq s \leq r}}\left\{h\left(\mathbf{x}\left(r_{f}\right), r_{f}\right)+\int_{r_{f}}^{r} g(\mathbf{x}(s), \mathbf{u}(s), s) d s\right\} \\
& =\min _{\substack{\mathbf{u}(s) \in \mathcal{U} \\
r_{f} \leq s \leq r+\Delta r}} \min _{\substack{\mathbf{u}(s) \in \mathcal{U} \\
r+\Delta r \leq s \leq r}}\left\{h\left(\mathbf{x}\left(r_{f}\right), r_{f}\right)+\int_{r_{f}}^{r+\Delta r} g(\mathbf{x}(s), \mathbf{u}(s), s) d s+\int_{r+\Delta r}^{r} g(\mathbf{x}(s), \mathbf{u}(s), s) d s\right\}
\end{aligned}
$$

where $\Delta r<0$. It is implicit here that at $r+\Delta r$, the system will be at state $\mathbf{x}(r+\Delta r)$. The double min operators arise from breaking the interval of integration into two separate intervals, namely, $r \in\left[r_{f}, r+\Delta r\right]$ and $r \in[r+\Delta r, r]$. From the definition of optimal cost-to-go function, we have the expression for $\left[r_{f}, r+\Delta r\right]$ given as $J^{*}(\mathbf{x}(r+\Delta r), r+\Delta r)=$ $\min _{\substack{\mathbf{u}(s) \in \mathcal{U} \\ r_{f} \leq s \leq r+\Delta r}} \int_{r_{f}}^{r+\Delta r} g(\mathbf{x}(s), \mathbf{u}(s), s) d s+h\left(\mathbf{x}\left(r_{f}\right), r_{f}\right)$. The principle of optimality requires that the continuation of an optimal trajectory is optimal, and thus,

$$
\begin{aligned}
J^{*}(\mathbf{x}(r), r) & =\min _{\substack{\mathbf{u}(s) \in \mathcal{U} \\
r+\Delta r \leq s \leq r}}\left\{\int_{r+\Delta r}^{r} g(\mathbf{x}(s), \mathbf{u}(s), s) d s+\min _{\substack{\mathbf{u}(s) \in \mathcal{U} \\
r_{f} \leq s \leq r+\Delta r}} \int_{r_{f}}^{r+\Delta r} g(\mathbf{x}(s), \mathbf{u}(s), s) d s+h\left(\mathbf{x}\left(r_{f}\right), r_{f}\right)\right\} \\
& =\min _{\substack{\mathbf{u}(s) \in \mathcal{U} \\
r+\Delta r \leq s \leq r}}\left\{\int_{r+\Delta r}^{r} g(\mathbf{x}(s), \mathbf{u}(s), s) d s+J^{*}(\mathbf{x}(r+\Delta r), r+\Delta r)\right\}
\end{aligned}
$$

Assuming $J^{*}(\mathbf{x}(r+\Delta r), r+\Delta r)$ has bounded second derivatives in both arguments, Taylor series expansion of this term $(\mathbf{x}(r), r)$ for (arbitrarily) small $\Delta r$ can be written as

$$
J^{*}(\mathbf{x}(r+\Delta r), r+\Delta r) \approx J^{*}(\mathbf{x}(r), r)+\left\{J_{r}^{*}(\mathbf{x}(r), r)+J_{\mathbf{x}}^{*}(\mathbf{x}(r), r) \mathbf{f}(\mathbf{x}(r), \mathbf{u}(r), r)\right\} \Delta r
$$

where $J_{r}^{*}(\mathbf{x}(r), r)=\frac{\partial J^{*}}{\partial r}(\mathbf{x}(r), r)$ and $J_{\mathbf{x}}^{*}(\mathbf{x}(r), r)=\frac{\partial J^{*}}{\partial \mathbf{x}}(\mathbf{x}(r), r)$. Substituting Eq. A.6 back into Eq. A.5 and letting $\Delta r \rightarrow 0$ yields

$$
J^{*}(\mathbf{x}(r), r)=\min _{\substack{\mathbf{u}(s) \in \mathcal{U} \\ r+\Delta r \leq s \leq r}}\left[-g(\mathbf{x}(r), \mathbf{u}(r), r) \Delta r+J^{*}(\mathbf{x}(r), r)+\left\{J_{r}^{*}(\mathbf{x}(r), r)+J_{\mathbf{x}}^{*}(\mathbf{x}(r), r) \mathbf{f}(\mathbf{x}(r), \mathbf{u}(r), r)\right\} \Delta r\right]
$$

Extracting out the terms that are independent of $\mathbf{u}(r)$, cancelling the term that appears on both sides, dividing by $\Delta r<0$, we finally have the HJB equation variant given as

$$
0=J_{r}^{*}(\mathbf{x}(r), r)+\max _{\mathbf{u}(s) \in \mathcal{U}}\left\{-g(\mathbf{x}(r), \mathbf{u}(r), r)+J_{\mathbf{x}}^{*}(\mathbf{x}(r), r) \mathbf{f}(\mathbf{x}(r), \mathbf{u}(r), r)\right\}
$$

The boundary condition can be represented as $J^{*}\left(\mathbf{x}\left(r_{f}\right), r_{f}\right)=h\left(\mathbf{x}\left(r_{f}\right), r_{f}\right)$ for $\left(\mathbf{x}\left(r_{f}\right), r_{f}\right)$ combinations that satisfy $\mathbf{m}\left(\mathbf{x}\left(r_{f}\right), r_{f}\right)=0$. 


\section{References}

[1] Guelman, M., "A Qualitative Study of Proportional Navigation,” IEEE Transactions on Aerospace and Electronic Systems, Vol. 7, No. 4, 1971, pp. 637-643. https://doi.org/10.1109/TAES.1971.310406.

[2] Becker, K., "Closed-Form Solution of Pure Proportion Navigation,” IEEE Transactions on Aerospace and Electronic Systems, Vol. 26, No. 3, 1990, pp. 526-533. https://doi.org/10.1109/7.106131

[3] Bryson, A. E., "Linear Feedback Solutions for Minimum Effort Interception, Rendezvous, and Soft Landing," AIAA Journal, Vol. 3, No. 8, 1965, pp. 1542-1544. https://doi.org/10.2514/3.3199

[4] Cottrell, R. G., “Optimal Intercept Guidance for Short-Range Tactical Missiles,” AIAA Journal, Vol. 9, No. 7, 1971, pp. 1414-1415. https://doi.org/10.2514/3.6369

[5] Jeon, I.-S., and Lee, J.-I., “Optimality of Proportional Navigation Based on Nonlinear Formulation,” IEEE Transactions on Aerospace and Electronic Systems, Vol. 46, No. 4, 2010, pp. 2051-2055. https://doi.org/10.1109/TAES.2010.5595614

[6] Cho, N., Kim, Y., and Shin, H.-S., "Generalized Formulation of Linear Nonquadratic Weighted Optimal Error Shaping Guidance Laws," Journal of Guidance, Control, and Dynamics, Vol. 43, No. 6, 2020, pp. 1143-1153. https://doi.org/10.2514/1.G004813.

[7] Guelman, M., and Shinar, J., “Optimal Guidance Law in the Plane,” Journal of Guidance, Control, and Dynamics, Vol. 7 , No. 4, 1984, pp. 471-476. https://doi.org/10.2514/3.19880

[8] Yang, C.-D., and Yeh, F.-B., “Optimal Proportional Navigation,” Journal of Guidance, Control, and Dynamics, Vol. 11, No. 4, 1988, pp. 375-377. https://doi.org/10.2514/3.20323.

[9] Lu, P., and Chavez, F. R., "Nonlinear Optimal Guidance," AIAA Guidance, Navigation, and Control Conference, Keystone, CO, USA, 2006. https://doi.org/10.2514/6.2006-6079

[10] Morgan, R. W., Tharp, H., and Vincent, T. L., "Minimum Energy Guidance for Aerodynamically Controlled Missiles," IEEE Transactions on Automatic Control, Vol. 56, No. 9, 2011, pp. 2026-2037. https://doi.org/10.1109/TAC.2011.2108619

[11] Chen, Z., and Shima, T., "Nonlinear Optimal Guidance for Intercepting a Stationary Target," Journal of Guidance, Control, and Dynamics, Vol. 42, No. 11, 2019, pp. 2418-2431. https://doi.org/10.2514/1.G004341

[12] Kalman, R. E., “When Is a Linear Control System Optimal?” Journal of Basic Engineering, Vol. 86, No. 1, 1963 , pp. 51-60. https://doi.org/10.1115/1.3653115

[13] Jameson, A., and Kreindler, E., "Inverse Problem of Linear Optimal Control," SIAM Journal on Control, Vol. 11, No. 1, 1973, pp. 1-19. https://doi.org/10.1137/0311001

[14] Kreindler, E., “Optimality of Proportional Navigation,” AIAA Journal, Vol. 11, No. 6, 1973, pp. 878-880. https://doi.org/10. $2514 / 3.50527$ 
[15] Lee, Y.-I., Kim, S.-H., and Tahk, M.-J., “Optimality of Linear Time-Varying Guidance for Impact Angle Control,” IEEE Transactions on Aerospace and Electronic Systems, Vol. 48, No. 4, 2012, pp. 2802-2817. https://doi.org/10.1109/TAES.2012. 6324662

[16] Song, T. L., and Um, T. Y., "Practical Guidance for Homing Missiles with Bearings-Only Measurements," IEEE Transactions on Aerospace and Electronic Systems, Vol. 32, No. 1, 1996, pp. 434-443. https://doi.org/10.1109/7.481284

[17] Kim, T.-H., Lee, C.-H., and Tahk, M.-J., “Time-to-go Polynomial Guidance with Trajectory Modulation for Observability Enhancement," IEEE Transactions on Aerospace and Electronic Systems, Vol. 49, No. 1, 2013, pp. 55-73. https://doi.org/10. 1109/TAES.2013.6404091

[18] Lee, C. H., Kim, T. H., and Tahk, M. J., "Biased PNG for Target Observability Enhancement Against Nonmaneuvering Targets," IEEE Transactions on Aerospace and Electronic Systems, Vol. 51, No. 1, 2015, pp. 2-17. https://doi.org/10.1109/TAES.2014. 120103

[19] Hull, D. G., Speyer, J. L., and Tseng, C. Y., "Maximum-Information Guidance for Homing Missiles," Journal of Guidance, Control, and Dynamics, Vol. 8, No. 4, 1985, pp. 494-497. https://doi.org/10.2514/3.20010

[20] Hull, D. G., Speyer, J. L., and Burris, D. B., "Linear-Quadratic Guidance Law for Dual Control of Homing Missiles,” Journal of Guidance, Control, and Dynamics, Vol. 13, No. 1, 1990, pp. 137-144. https://doi.org/10.2514/3.20527

[21] He, S., Shin, H.-S., Ra, W.-S., and Tsourdos, A., “Observability-Enhancement Optimal Guidance Law,” 2019 Workshop on Research, Education and Development of Unmanned Aerial Systems (RED UAS), Cranfield, UK, 2019. https://doi.org/10.1109/ REDUAS47371.2019.8999706 
2021-07-21

\section{Inverse optimality of pure proportional navigation guidance for stationary targets}

\section{Lee, Seokwon}

American Institute of Aeronautics and Astronautics

Lee S, Cho N. (2021) Inverse optimality of pure proportional navigation guidance for stationary targets. Journal of Guidance, Control, and Dynamics, Available online 21 July 2021.

https://doi.org/10.2514/1.G006018

Downloaded from Cranfield Library Services E-Repository 